\title{
Extrapolating Back in Time to Our Off-Planet Ancestors
}

By John F. Caddy

Introduction- Trying to guess what happened in the distant past and where we come from, requires a juggling of unknowns, and from most points of view that is what you are going to read here. At the same time, as we accumulate more information, it begins to be clear that the few clues left for us exclude certain possibilities, and point to constantly occurring processes such as Syntropy being involved. It's always been a mystery to me why for example, physical science is enamoured of the concept of Entropy. Perhaps it is because a decline in structural complexity with time describes well the degradation of our world over time under the dominant philosophy of materialism. Its opposing concept, Syntropy, implies an increase in complexity, brought about by a mysterious force apparently acting from the future, and this process is less easily fitted into a Newtonian universe. Syntropy, or 'the law of creative connecting' is defined by the Free Dictionary as the force used by the universe to move us towards a brighter future, and its action is described by vocabularies such as: 'The will to become', 'Intent', 'Evolution'. These terms seem to describe the origin of life and intelligence, but Syntropy has been largely shunned by science.

One of the most effective mechanisms of syntropy is that for past ages, intelligent life forms have been arriving here, and still are coming to our planet from somewhere else in the cosmos, using their discoveries to speed up our evolution.

GJHSS-H Classification: FOR Code: 049999

Strictly as per the compliance and regulations of:

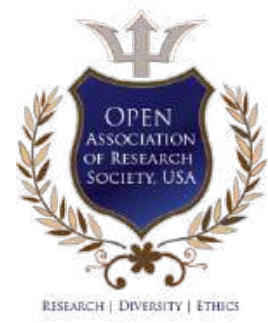

(C) 2020. John F. Caddy. This is a research/review paper, distributed under the terms of the Creative Commons AttributionNoncommercial 3.0 Unported License http://creativecommons.org/licenses/by-nc/3.0/), permitting all non-commercial use, distribution, and reproduction in any medium, provided the orig inal work is properly cited. 


\title{
Extrapolating Back in Time to Our Off-Planet Ancestors
}

\author{
John F. Caddy
}

\begin{abstract}
'We are suspended in a continuous present in which anything could, and likely did, happen'
\end{abstract}

\section{INTRODUCTION}

( rying to guess what happened in the distant past and where we come from, requires a juggling of unknowns, and from most points of view that is what you are going to read here. At the same time, as we accumulate more information, it begins to be clear that the few clues left for us exclude certain possibilities, and point to constantly occurring processes such as Syntropy being involved. It's always been a mystery to me why for example, physical science is enamoured of the concept of Entropy. Perhaps it is because a decline in structural complexity with time describes well the degradation of our world over time under the dominant philosophy of materialism. Its opposing concept, Syntropy, implies an increase in complexity, brought about by a mysterious force apparently acting from the future, and this process is less easily fitted into a Newtonian universe. Syntropy, or 'the law of creative connecting' is defined by the Free Dictionary as the force used by the universe to move us towards a brighter future, and its action is described by vocabularies such as: 'The will to become', 'Intent', 'Evolution'. These terms seem to describe the origin of life and intelligence, but Syntropy has been largely shunned by science.

One of the most effective mechanisms of syntropy is that for past ages, intelligent life forms have been arriving here, and still are coming to our planet from somewhere else in the cosmos, using their discoveries to speed up our evolution. By definition, Life is an integral feature of the Universe, just as Intelligence is also, and we should not base our arguments on the assumption that events are always a consequence of random processes which are integral component of entropy.

It is natural to question how our species got to where it is today, and this is my personal attempt to resolve this question. It is still premature to assume that a deliberate introduction of life forms onto this planet occurred soon after the surface of our planet cooled sufficiently, but there are serious motives not to automatically assume that Life was generated here. Some of these are:

Author:Ph.D. e-mail: jfcaddy1@yahoo.co.uk
- The fundamental role of the highly complex molecule DNA in all life forms examined so far, is probably not compatible with its evolution here. In the opinion of one of its discoverers, Francis Crick ${ }^{1}$, it would require a period longer than the lifespan of Earth so far (4.54 billion years) to achieve this by random processes; a conclusion shared by theoretical calculations ${ }^{2}$, while some estimates of when life began here suggest it began before 3.9 billion years.

- The logical corollary to this statement is that living ecosystems and societies of intelligent creatures ${ }^{1}$ existed elsewhere before Earth was formed. Simple life forms either arrived on Earth by deliberate introduction (implying a role for intelligent life forms who evolved elsewhere before us), or by random panspermia from life forms transported into space by meteorite strikes on other planets; e.g. Mars, where a number of factors suggest life was present before it appeared on Earth, or were 'spontaneously generated here; the current dominant belief by science to be the case.

- The existence of intelligent life forms on Mars millions of years ago before drastic climatic changes wiped out free bodies of water and a breathable atmosphere, is a reasonable deduction from NASA imagery of surface structures that it would be difficult to ascribe to random geological processes. . $^{3,4}$

- Life forms may have reached Earth by diffusion from other sources, such as the planet whose destruction gave rise to the asteroid belt ${ }^{4}$, or by directed panspermia from outside the solar system.

Under this last option we need to extrapolate backwards in time to envisage a point of initiation for a program of directed panspermia by extraterrestrials coming from light years away, and this is the main possibility driving this speculation. I am personally convinced that in comparison with our 300-400 years of recent scientific evolution, that ETs may have been scientifically competent for thousands or even millions of years. Those sceptics who deny the existence of UFO's because they are afraid that super-intelligent beings will take over this planet, are comforted by Einstein's discovery that the limit to the speed of light have prevented interstellar voyages that began from light years away. To suggest that ET could not find a way round this roadblock in thousands of years of research, 
is not an intelligent reason for denying thousands of observations annually of their presence around this planet. Many thousands of years in the past our ancestors were less easily fooled and recorded humanoid figures in space suits in their rock engravings! At that early time and even today, technically advanced ETs could have taken over this planet without a problem. Continuing to deny the reality of their presence is pure xenophobia and blocks our future development.

Many of these advanced life forms visiting here (some 85 species have been identified by associations of ufologists ${ }^{5}$ ), are basically similar in external bodily structure and features to ourselves. In fact, a large number of extraterrestrial species identified by observers show a surprisingly limited diversity in external anatomy ${ }^{5}$. The hominid body form is the most common, although this does not exclude ETs from facially resembling non-mammalian forms, while still showing an overall hominid body structure.

A reasonable deduction from this observation is that selection for a hominid form has occurred elsewhere than on this planet on numerous occasions: surprising evidence for convergent evolution if they were totally independent genetically! Could the common hominid modality have been more logically achieved if a common thread of evolution were accepted ? A hominid modality is a mechanically convenient framework, ensuring easy manipulation of objects by the hands and a vertical posture allowing binocular vision from an above-ground position, so proposing that some structural elements have been inherited in common across cosmic distances is not out of the question.

As discussed in the following, there also appears to be some justification for assuming close similarities in cellular and reproductive structures to ourselves, although evidence for the existence of cloned or advanced robotic forms among ETs cannot be excluded.

Relevant arguments for these small-scale physiological similarities are:

- That if panspermia played a key role in transmission of life forms between an original and a distant planet, it seems likely that a similar genomic structure, and some genetic components for intelligence and overall anatomy were also conserved.

- Evidence for this last point comes from a significant component of recent close encounters with ETs involving 'kidnapping'6 of humans; their transportation to ET craft for extraction of cellular tissues, and their return to Earth largely unharmed physically. From subsequent hypnotic reconstruction of the memories of the abductees ${ }^{7}$, (memories deliberately obfuscated by the ET's involved), it is evident that a sampling of reproductive tissues or gametes has often occurred $^{6}$.

- This suggests that searching for useful genetic material was the main objective of the ETs for kidnapping humans, and has involved thousands of humans, most of whom have had their awareness of this event eliminated. Repetitive sampling of the same individuals or family lines also seems to have taken place. Individuals were marked by a subepidermal tag of off-planet material ${ }^{8}$. This suggests that an attempt was being made to track particular genomes in the human population, for the same reason we humans track individual animals in game parks.

The main purpose of this note is not to protest at our loss of choice in this matter, however disturbing it is to be treated as an inferior or experimental species (perhaps not for the first time in our evolution?). What I am trying to clarify here is a hypothesis that explains the apparent convergence between human and ET genetic structures and appearances.

\section{il. New Postulates}

Synthesis of DNA by natural evolutionary processes has been regarded as close to impossible in much less than a billion years due to its molecular complexity ${ }^{1,8}$. We start our reasoning then from a commonly-quoted estimate that the Earth was formed some 4.54 billion years ago as a ball of incandescent lava, A commonly-quoted opinion is that 'several hundreds of millions of years later, surface water was formed'. The synthesis of DNA, if it was formed naturally on this planet, should already have begun a hundred million years after planetary birth but was necessarily achieved before 3.7 billion years ago when the first fossils were formed ${ }^{10}$. On the face of it, this leaves roughly 0.8 billion years for DNA to be synthesized, multicellular organisms to have evolved, and for them to have formed fossils, by some 0.8 billion years after the birth of this planet. To me, this appears to be a tight time frame for all of the following events to occur: the synthesis of DNA in nature, the evolution of single-celled then metazoan life forms, and sufficient biomass accumulated to be visible in fossilized form.

In the opinion of Crick and Orgel ${ }^{1}$, this simplistic type of calculation must imply that DNA originated elsewhere than on this planet and microscopic organisms were probably brought here by visiting ET's who evolved before us. This is seen as close to a proof of directed panspermia by advanced ET's as we are likely to get until informed otherwise by 'antique' ETs who started their technological evolution much earlier than we did. They may have records of our earlier evolutionary stages that this note is attempting to reconstruct. 
What could be their motivation for persistent visits to our planet? Some suggestions from the literature are:

- They actually live here: (the deep oceans, Antarctica and deep underground locations have been speculated to host ET home bases), and the reader is invited to search the internet for more clues, with particular attention to Antarctica ${ }^{11}$;

- They visit Earth regularly to monitor their long-term research programs;

- Secret residence agreements have been established with various governments (again, do your own searches to decide on the validity of this possibility), in exchange perhaps for access to high technologies;

- Has the evolution of high technology in the 75 years since the second world war occurred too rapidly if we assume no extraterrestrial assistance took place?

- ET's are here to prepare the planet for future colonization by their species, after modifying their own genome with sampled human DNA to preadapt it to Earth conditions.

Some specific issues have arisen recently that relate to directed panspermia and the technological issues involved. These are:

- Could sharing a common origin through directed panspermia, be the reason for the compatibility of genome elements between different hominid forms, making the 'borrowing' of our gametes of immediate interest?

Recent balloon samplings of the stratosphere some $17 \mathrm{~km}$ above the Earth by Dr Milton Wainwright have captured fragments of microscopic extraterrestrial life forms, but also a small sphere of an exotic alloy (titanium and vanadium were components mentioned), that contained biological organisms ${ }^{12}$. It may be relevant that various exotic alloys called 'shape-memory alloys' are now used in surgery to construct surgical staples which open and close automatically when exposed to different ambient temperatures ${ }^{13}$. A container made of such an alloy would be an ideal mechanism for directed panspermia: it could be designed to open upon reaching warmer temperatures at the Earth's surface if space-borne panspermia were being planned.

There is evidence that a significant number of different species of extraterrestrials ${ }^{14}$ have been visiting Earth; many of which have a hominid body structure, although some features may resemble amphibian, reptilian or mammalian forms found on Earth. Canadian former minister of defence, Paul Hellyer referred to a large number of different ET species visiting us, and somewhat smaller inventories have been made elsewhere. Locating a reliable source of information is not easy since the total number of ET species visiting Earth, although apparently large, varies dramatically with different authors. Thus, the exact number of ET species coming here or even living here, is still not accurately defined. However, the apparent externality of hominid forms, presumably coming from different planets, is the key factor. This seems evidence for a sequence of events promoting the diffusion of a body structure externally similar to humans, and this is the issue focussed on in the following.

As mentioned earlier, ${ }^{6}$ the frequent 'kidnapping' of humans is well documented, and the extraction of tissue samples before returning the victims largely unharmed to Earth seemed the main objective of the ET. The victim's conscious memory of this event was erased, but on personal recollection, notable gaps in their individual passage of time were evident. There is even a repeated rumour that this practice was supported by a negotiated agreement between a species of ET and the government of an important country. Rather than cite individual publications for such assertions that are anyway rendered uncertain by the secrecy that envelops the subject of ETs, it is suggested that the reader make internet searches to locate the isolated pieces of information which are freely available on the web. They should then arrive at their own decisions on the validity of the hypotheses discussed here.

Regressive hypnosis ${ }^{7}$ has been used by psychiatrists to access the supressed memories of some of those kidnapped victims, and elements of these events have emerged that show much in common between the individuals so affected. As noted above, there appear to have been repeated samplings of the same individuals, perhaps aided in their identification by the ETs marking them with granules of foreign material embedded sub-epidermally ${ }^{16}$. These granules apparently consist of off-planet materials that were identified in X-rays and removed surgically. They do not stimulate allergic reactions in human tissues, but neural linkages are formed in adjacent tissues.

The motivation for this ET activity can be various. However, travelling far back in time to the Stone Age and reading the chromosomes of other fossil hominids, has unearthed genetic evidence for crossfertilization between different human species on Earth ${ }^{17}$. Thus, genetic material from different hominid species who evolved in different environments on Earth have contributed to our genome. One could conventionally conclude that this occurred by hybridization, due to encounters in nature between different human species, but that is an unproven assumption.

With respect to hypotheses of the colonization of the Earth by intelligent species, I mention again several obvious possibilities for their persistent presence near our planet:

- A common opinion is that the 'colonists' were members of the crew(s) of the ET ship(s) that visited Earth, perhaps for research purposes, who then decided to colonize this planet? In other words, this 
hypothesis assumes we are their direct descendants. There may be an element of truth in this idea, but not as direct descendants. For example, where did the other fauna and flora they needed to live on come from? Did they bring with them their complete ecosystem?

- This first hypothesis has the serious drawback that a high proportion of the elements of human DNA are shared with many other members of our planetary fauna (up to $96 \%$ of genes for some great apes are also common to human beings ${ }^{18}$ ), suggesting a common evolutionary origin links us to them. Even for terrestrial insects the genes shared with us approach $60 \%$. Thus we can exclude categorically that we are the direct descendants of ET colonists, even if we may have received some elements of their genome by some gene splicing procedure.

- That almost all fauna and flora on Earth can be traced taxonomically and genetically to a common single-celled ancestral form, seems to imply that evolution of our ecosystem can be traced back to a single starting point. This suggests that life began here once only; either by 'inoculation' from offplanet, or by a single event of creation, such as the first (and only?) spontaneous evolution on Earth of an ancestral organism, supposedly equipped simultaneously with DNA (the so-called 'Big Birth'?) If this happened, it was certainly not replicated in the following 4 billion years, suggesting that 'the spontaneous generation of life' is a very improbable event.

Although it is certain that overall, ET genomes are different from ours, their chromosomal structure and gene components must be similar in pattern to ours, otherwise why would they kidnap humans to sample our DNA? Perhaps their genomes were originally even closer to ours but were modified by advanced genetic manipulations or cloning to facilitate space-going? Perhaps losses of the key genetic components from 'primitive hominids' during past artificial reproduction need replacing?

\section{ili. Past Hybidization of Humans?}

Several accounts support the idea that hybridisation and/or gene splicing from ET hominid sources has long been within the capabilities of some advanced ET's who visited Earth. Evidence coming from genetic studies ${ }^{19}$ is compatible with the idea that gene modification and selective breeding of humans were carried out some 20,000 years ago or more by advanced (ET) civilizations. Recent genetic studies suggest that an unusually large number of mutations affecting human brain structure and function occurred around that time ${ }^{20}$.

At least two ET species, the Anunnaki ${ }^{19}$, and ETs coming from the Pleiades ${ }^{20}$, have been postulated to have undertaken gene transplants from their genome to that of pre-humans. The speculated benign objective of the Anunnaki interventions was to promote rational behaviour in a future component of a multi-species society, or perhaps less idealistically, to 'tame' a slave population needed for manual work ${ }^{21}$ ?

One incidental result of interventions by the Anunnaki was to induce in humans a dominant (leftbrained) mentality, using as test subjects individuals from small cooperating bands of hunter-gatherers who, through right-brain dominance, were given to artistic expression (see the Cro-Magnon engravings from 20-30 thousand years ago). The modified individuals may have better fitted into a complex, vertically-structured society which emphasized the division between rulers and workers/slaves ${ }^{24}$. Such an adaption was also well-suited for organized warfare using human serfs as combatants in Anunnaki internecine feuding. This strategy of mass conflict may also have been useful as a dynamic for reducing competition from fast-breeding humans with their 'masters'. Separate societies of short-lived humans could have been transported to different regions of the world by their long-lived Anunnaki masters, following their 'Tower of Babel Strategy'. This strategy encouraged diversification by the separated human groups in language, religion and culture, thus discouraging their mutual cooperation in warfare against their Anunnaki masters ${ }^{24}$

As noted, recent discoveries have identified Neanderthal and Denisovian components in the DNA of modern humans ${ }^{25}$. It is widely assumed that this mixture was a result of random sexual encounters between bands of different terrestrial hominids. That a degree of genome mixing might have been a result of selective breeding or gene splicing from other hominids by Anunnaki or other ET species in creating modern humans, has apparently not been considered so far.

The description by $\operatorname{Sitchin}^{19}$ of genetic intervention was focussed on the introduction of limited components from the Anunnaki genome to humans. These interventions emphasized docility, and in contrast to hunter-gatherers, a readiness to accept orders from above. This ensured that subservient human populations were kept manageable despite a much faster rate of population growth than for the Anunnaki and their hybrids.

That there was also direct hybridization between humans and Anunnaki was contended by $\operatorname{Sitchin}^{19}$, and skeletons of the resulting giant forms have been discovered throughout the world, but so far these have not been treated as a separate species of humans by anthropologists ${ }^{27}$, In fact the relicts of ET visitors and their hybrids have been played down scientifically, to avoid reviving a traditional fear of giants. The role of giant hominids in pre-Biblical, Sumerian and predynastic Egypt, is suggested by an apparent association of these forms with the ruling caste 
responsible for megalithic constructions. It is tempting to see them as the equivalent of the Biblical era giants, the Nephilim, mentioned in the Book of Enoch ${ }^{30}$. Perhaps due to their low fertility and long lifespan, these hybrid forms engaged in warfare between themselves, using genetically-modified humans as soldiers? There is accumulated evidence that they even used atomic weapons ${ }^{31}$, perhaps initially in feuds between Anunnaki families, but later to suppress the development of independent societies run by humans (such as Sodom and Gomorrha). From Sitchin's translations of ancient Sumerian, the human obsession with reproduction alarmed the less fecund and long-lived ET species and their hybrids, so that their future loss of dominance was guaranteed.

\section{Hominid Origins}

In my earlier book ${ }^{22}$ I pointed out that it is quite possible that prior to their demise some 65 million years ago, a reptilian civilization evolved, and could have reached a high level, perhaps even attaining space flight. The idea of a dinosaur culture seems one of the few reasonable explanations for the discovery of artefacts in steel and aluminium found in Jurassic coal seams $^{32}$. The suggestion was also made that intelligent reptilians lived underground, and this stratagem could have allowed them to survive bombardment from space. Such an adaptation would later be useful for living on planets without breathable atmospheres, where they would be less vulnerable underground to space debris or deliberate attacks with diverted meteors.

Although primitive mammals had already evolved prior to the disappearance of giant dinosaurs, I have used 65 million years as the time frame needed for such primitive forms to reach intelligence and a technological-based reptilian society. This might give us one admittedly very uncertain estimate of the time frame required for hominid-structured reptilians to become high-tech and potentially space-going, and clearly the Jurassic and the preceding Triassic provided ample time for social species of bipedal dinosaurs to rise to intelligence.

\section{A Conclusion of Sorts....}

I assume that earlier in its 10 billion year history, the cosmos gave rise to the first intelligent life forms, perhaps 5-7 billion years ago. If these 'Primogenitors' still survive in biological form, they will have evolved so far in advance of ourselves as to be almost unimaginable to the human mind.

A theory that is not implausible, given the above evidence for directed panspermia, is to assume that life forms evolved on the first cool water-bearing planet to arise in the cosmos billions of years ago. This 'birth planet' must have had some dry land and been stable for a significantly longer period than experienced so far by planet Earth, thus allowing for the evolution of DNA. Assume then, that 'The Big Birth' that eventually gave rise to life forms, began with unicellular forms and sizes, and that genetic information was first coded in RNA and protein structure. Eventually single and multiple-celled organisms arose through evolution with simple forms of spiral DNA. Multicellular forms, over the long term, eventually gave rise to an intelligent hominid species, which in time also achieved a technically-competent society, and eventually space flight.

The following figure then offers a suggestion as to how successive colonization of suitable planets, possibly aided later by directed panspermia, led to them sharing common elements of their original bodily structure and global ecosystems with colonized planets. These could have including the diffusion of a hominid anatomy, and key common elements in their genome, although subsequent genetic diversification would have been widespread, if not controlled.

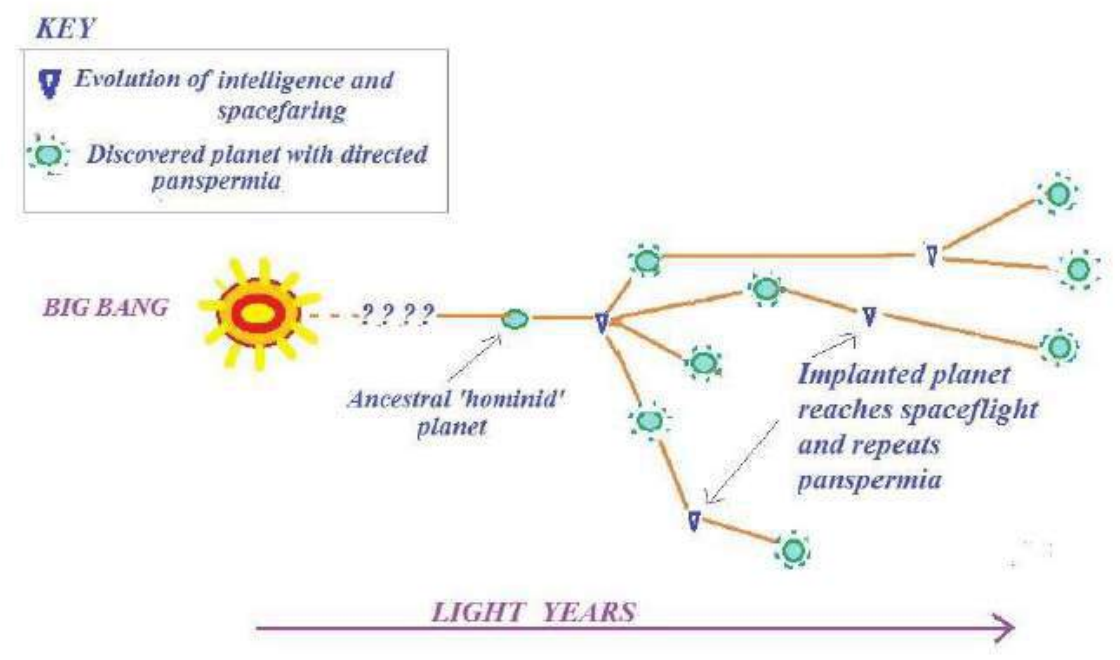


Fig 1. Conceptualizing a possible evolutionary sequence followed by a hominid form resembling the ancestral home genotype, starting from an ancestral planet where it first evolved, and where space flight was first discovered. It is assumed that suitable planets were either colonized and evolutionary adaptation occurred, or a form of directed or random panspermia took place, eventually leading to evolution of variants on the hominid design and ecosystems.

Going back to the early diffusion through the cosmos of the 'Primogenitor species' in search of waterbearing planets similar to their first human world of origin, we might assume that on early-discovered planets, simple unicellular or metazoan organisms from their home world were introduced accidentally. However, when the advantages of a more deliberate, rapid and reasoned process for the future colonization of these worlds became evident, a tradition may have arisen of introducing useful life forms from their original home planet to create supporting ecosystems. In the course of time these secondary ecosystems came to resemble the original ecosystem that generated their own species. These 'copied' ecosystems could have given rise either to descendants of the colonists, or eventually to one or more intelligent species in hominid form, evolved from the common genotypes that were inoculated by directed panspermia. Given that evolution on these colonized worlds was based on the genomes of the original world, it would not be surprising if some directed evolution occurred, perhaps partly programmed by the 'silent' components of 'Junk DNA' which forms much of our chromosomes and still remains to be interpreted ${ }^{33}$. This may have directed evolution so that the forms that arose later, still retained some similarities to the fauna and flora on the planet of the 'Originators'?

\section{The Time Frame for the Evolution of INTELLIGENCE}

One of the problems for our short-lived species in discussing this topic, is that it is hard for us to imagine implementing a strategy of planetary colonization involving a time frame of millions of years to develop a liveable planetary ecosystem by terraforming from scratch. One could counter that our intelligent species predecessors had life spans multiples of ours, hence they would have been used to thinking far ahead. Or it could be that so many planetary prospects were found during exploration that developing a seeding strategy was more feasible than colonization over the short term? More practically perhaps, the obvious solution for speeding up evolution would be to transport to lifeless planets the germ cells from their world of plants and then animals, to speed colonization and further evolution - probably on colonized planets these organisms were first grown within artificial enclosures then released into the wild at favourable locations? This process is nonetheless now being discussed by future colonists of planet Mars.

Finally, from a socio-political perspective, we might assume that our predecessors went through a stage of warfare such as we are still embroiled in. From this, they may have concluded logically, by experience, or by negotiation, that planetary civilizations are too vulnerable to bombardment from space and that peaceful multi-species coexistence is the only option. Multiple rumours have suggested that our species and planet are protected from unscrupulous colonization by such an association similar to a space-going UN?

Faced with a close to infinite selection of planets to colonize or terraform, there is less need for competition for planetary surfaces, and space-going artificial surfaces inside for example, large asteroids, are now the subject of science fiction. 'Terraforming' a lifeless planet requires motivation, but also a great commitment over many generations: future generations whose requirements must be placed in the forefront. Could it be that the basic motivation of ETs was to increase the abundance of intelligent life in the cosmos, and by applying syntropy on a large scale, make the cosmos a better place to live in? Oh I agree, that's even more farfetched than the rest of this article, but I've reached the end of my arguments, and what else is there left to say?

\section{Selected References}

1. Crick, F. and H. Orgel, L. E. (1973). "Directed panspermia". Icarus. 19 (3): 341-346.

2. Anon. (2017). Strange Structures on Mars Connected To The Sphinx and Pyramids Of Gizeh? July 8, 2017. Disclose TV.

3. Hoagland R.C. (2014). Chinese Lunar Images. Coast to Coast. 22/4/2014.

4. Imdahl, C. (2018). Researchers have found remarkable remains of destroyed planets in space. In: Business Insider.

5. Jones, K. (2016). Top 10 different alien species on Earth. Proof of Alien Life. (Proofofalien.com 03/13/2016).

6. Robinson, S.P. (1998). UFO's, Aliens and Alien abductions. http://www.niu.edu/newsplace/ nnaliens.html.

7. Booth, B. (2017). 1961 - The Hills: abducted by aliens. Liveaboutdotcom (https://liveabout.com).

8. Anon. (2018). Alien Implants. In: www.alieneverything-you-want-to-know.com

9. Yirka, Bob. (2013). Researchers use Moore's Law to calculate that life began before Earth existed. https://phys. org/news/2013-04-law-life-began-earth. html.April 18, 2013.

10. Anon. The oldest Fossils ever found! YouTube 02/09/2016 
11. Veall, W.J. (2017).Tamils and Sumerians. Among the first to Reach Australia and Antarctica? PART I. Ancient Origins 6 Dec. 2017.

12. McClure, K. and C. Kloetzke (2017). Is Earth Being Seeded with DNA-Filled Metallic Spheres from Space? https://thefieldreportscom.wordpress. com/2017/08/29/is-earth-being-seeded-with-dnafilled-metallic-spheres-from-space/

13. Bahraminasab, M and B. Bin Sahari. $(2912+)$. NiTi shape memory alloys, promising materials in orthopedic applications. Chapter 10: (https://cdn. intechopen.com/pdfs/44018.pdf).

14. Anon. Known types of Aliens and Races. The Watcher Files. (thewatcherfiles.com/alien_races. html)

15. Anon. Removal of an Alien Implant. YouTube 04/05/2009.

16. Lovegren, S. (2005). Chimps, humans $96 \%$ the same, gene study finds. Natural Geographic Magazine.

17. Ancient Aliens. Alien messages in our DNA. (Session 9)| History. YouTube 02/07/2019.

18. Vanderhaegen Lab. (2018). How did human brains get so large? VIB rep ort Holland; 31 May 2018.

19. Sitchin, Zecharia. (1976). The Twelfth Planet. HarperCollins Publisher, New York.

20. Fenton, D. (2018) 'Hybrid Humans: Scientific Evidence of Our 800,000-Year Old Alien Legacy'.

21. Barras, Colin. (2018). Survival of the tamest. New Scientist, 24 February, 2018.

22. Caddy, J.F. (2019). Did extraterrestrials bring us to intelligence on our planet? Xlibris Publications, $123 \mathrm{p}$.

23. Marzulli, L.A. (2016). New DNA Testing on 2,000Year-Old Elongated Paracas Skulls Changes Known History. Ancient Origins, 23 JULY, 2016

24. Tellinger, Michael. (2005). Slave species of the gods: the secret history of the Anunnaki and their mission on Earth. Bear and Company.

25. Anon. Modern humans may have interbred with Neanderthals and Denisovians. YouTube 21/12/2009.

26. Gigal. (2018). A giant Pharaoh was discovered according to Science? (htpps://www.gigalinsights).

27. Little, G. (2014). The truth about giant skeletons in American Indian mounds, and the Smithsonian cover-up. AP Magazine, Sat. 28 Jun 2014.

28. Book of Enoch - Wikipedia. https://en.wikipedia. org/wiki/Book_of_Enoch.

29. Anon. (2014). Physicist Claims Evidence Ancient Nuclear Explosions Ended Life On Mars. Inquisitr November 21, 2014.

30. Gaia (2018). Powerful Evidence of Nuclear Wars in Ancient Times. Gaia April 18, 2018.

31. Steiger, Rod. (Dec. 2007). Exists numerous evidence of prehistoric nuclear war. The Canadian Website.
32. 5 Mysterious artifacts no one can explain. YouTube 02/06/2015.

33. Is most of our DNA junk? YouTube. 24/05/2019. 